\title{
Can You Outthink a Smart Machine?
}

\author{
By Ed Hess
}

The title of this article is the existential question that artificial intelligence (AI) will likely raise for many human knowledge workers, especially those in the financial industry. Technology is not new in the financial industry; it fueled the development of hedge funds and underlies most sophisticated data analysis and trading systems. What is different now is the possibility that technology itself will be able to learn and adapt, as evidenced by the performance of the Google DeepMind AI technology in beating master go player Lee Sedol 4-1 in a recent best-offive match. Deep learning is a transformative possibility that is being actively pursued by Google, Microsoft, IBM, and Baidu, among others, along with many privately funded companies.

The consensus view over the last few years has been that technology in the next 5 to 15 years is going to automate a lot of tasks that human knowledge workers perform, aside from the higher-order type of thinking (critical, innovative, and creative thinking). Automation will move into numerous professions, including accounting, science, architecture, law, medicine, and business.

Many of us professional knowledge workers have taken solace in the prediction that we will still be needed to do the complex thinking (e.g., critical, innovative). That solace is somewhat misplaced, however, because the science is clear that most individuals are suboptimal performers in those areas. As discussed in my 2014 book Learn or Die: Using Science to Build a Leading-Edge Learning Organization, we humans are basically reflexive confirmation and affirmation thinkers. By that I mean that cognitively, we generally seek to confirm what we already believe, and emotionally, we tend to seek affirmation by defending those beliefs and our views of ourselves. To compete, stay relevant, and add value in the age of smart machines, most of

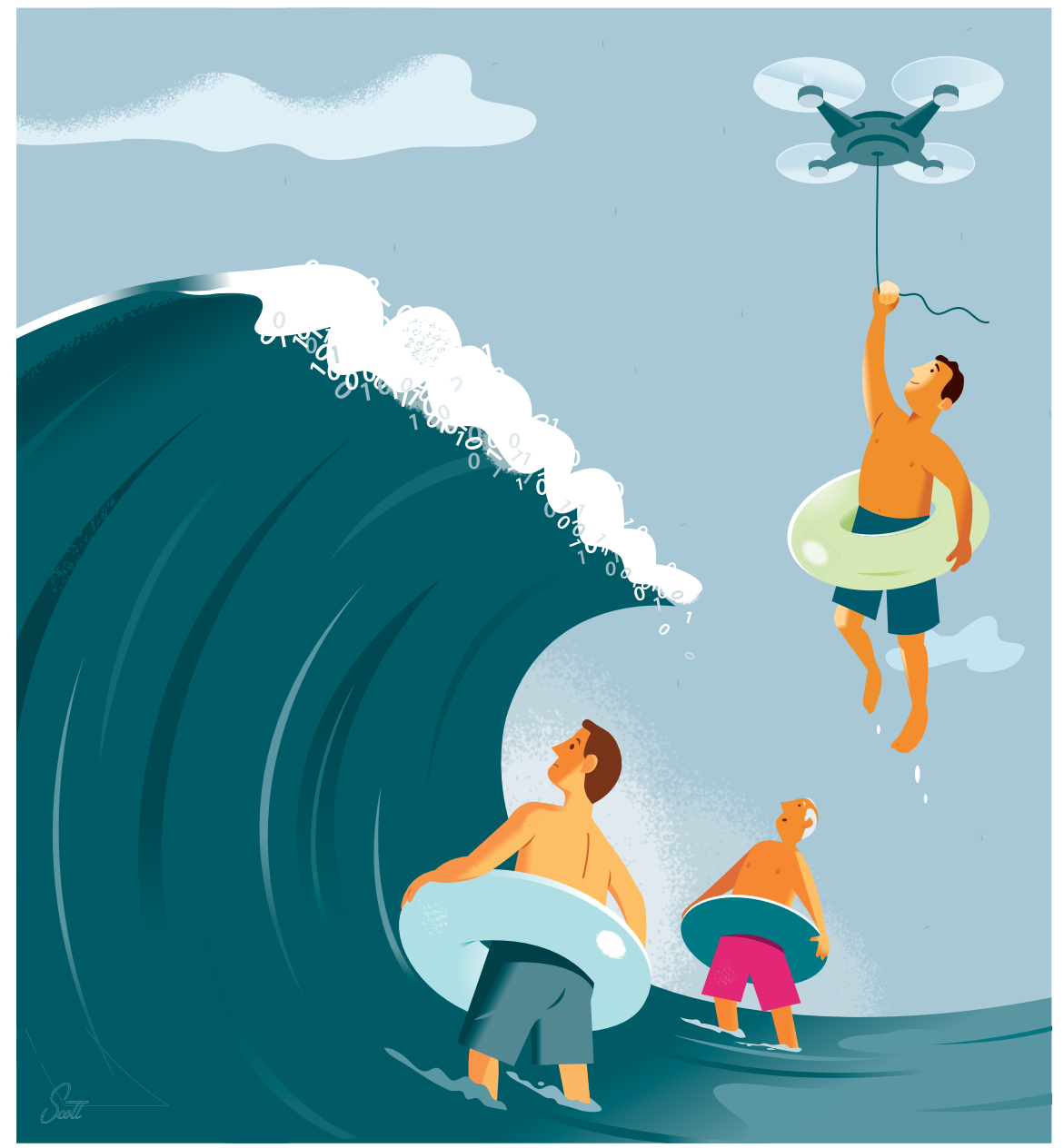

us will have to take our thinking, listening, engaging, and collaborating game to a much higher level. For many, that will require a new mindset-unlearning and relearning.

Cognitive science states that it is very hard for any of us to overcome our thinking limitations by ourselves. We need the help of others to do that. Higher-order thinking requires thinking with others. I have spent the past five years researching high-performance learning organizations that have created internal systems designed to take human thinking performance to its highest levels-firms such as Bridgewater Associates, Google, Pixar, IDEO, and the special operation forces of the US Armed Forces. Thinking with others requires the right type of work environment and the rigorous use of best learning processes.

The right environment is one that mitigates the two greatest learning inhibitors-ego and fear. We have to be willing to accept the science of learning, which clearly states that we are suboptimal learners, and be willing to subject our thinking to constant stress testing by others and our performance to frequent review. That can only happen in an environment of trust and psychological safety that is as devoid as humanly possible of politics and interpersonal competition.

Bridgewater Associates is the most advanced learning organization I have studied because it has confronted ego 
and fear head on. It seeks to transform the human tendency to feel insecure when one's thinking is challenged to a tendency to feel insecure when one's thinking is not challenged. It is public knowledge that Bridgewater Associates is engaged in taking its thinking to even higher levels with hiring more expertise in the area of AI. To me, that is a big signal. If Bridgewater is seeking to take its already high thinking and learning capability to an even higher level, should you?

What does this mean for CFA charterholders? I don't know. My educated guess is that every charterholder will need to take his or her thinking, listening, and collaboration skills to a higher level, and that requires quieting one's ego and developing one's emotional intelligence. The ability to manage self-one's thinking, listening, and relating - may become as important as one's financial analysis acumen.

AI and deep learning also raise the issue of what type of financial analysis charterholders will be needed to do that technology can't do well. In part, this depends on how technology is going to transform businesses. What could the organization of the future look like? Technological advances (including the Internet of Things, big data, and AI) will likely transform how most businesses are staffed, operated, and managed. The organization of the future will likely be staffed by some combination of smart robots, smart machines, and humans. Technology could well commodify operational excellence, making innovation the key value-creation differentiator for many businesses.

That would mean that organizational and individual learning, as well as adaptability and responsiveness to multiple converging forces of change, will become strategic necessities. Being able to assess organizational capabilities, technology capabilities, and the innovation pipeline-as well as company portfolios - may well become key skills for finance professionals. This sort of assessment requires more than just numbers; for example, assessing the emotional environment of an organization and the effectiveness of its internal system to drive learning behaviors becomes relevant.

Financial analysts need not feel singled out. These questions apply equally to other professions underpinned by analytical thinking. All will have to answer the question, "What can we do well that technology can't do well?" We are on the verge of a likely technology tsunami that is going to create immense opportunities and challenges for us humans that will require many of us to place more emphasis on developing our emotional intelligence and our creative, innovative thinking capabilities. Our only sustainable competitive advantage may well be learning faster than the technology advances.

Ed Hess is professor of business administration and Batten Executive-in-Residence at the Darden Graduate School of Business, University of Virginia. He is the author of 12 books focusing on growth, innovation and learning systems, and cultures and processes.

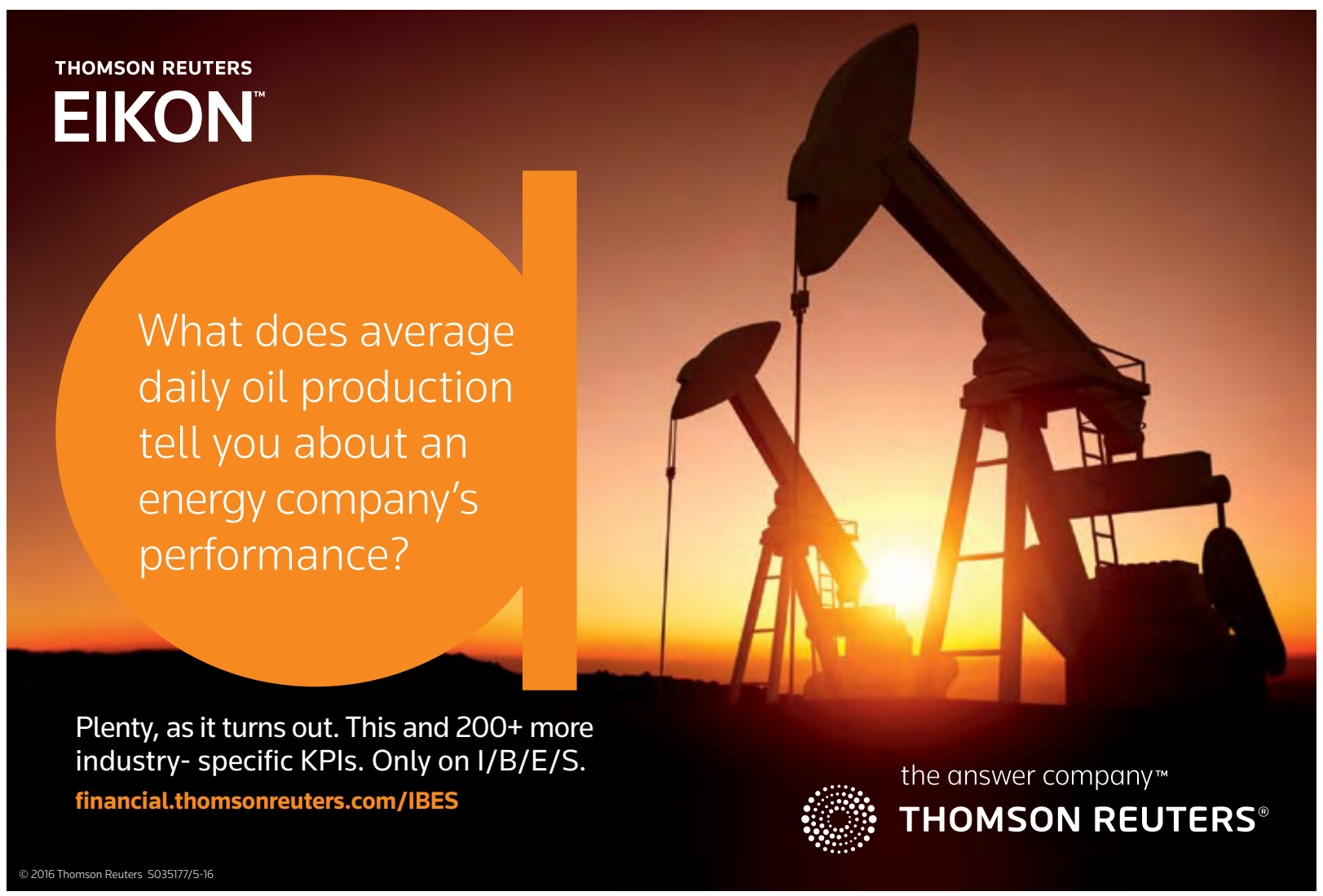

